\title{
ARTICLE
}

Received 9 Jul 2014 | Accepted 8 Oct 2014 | Published 25 Nov 2014

DOI: $10.1038 /$ ncomms 6517

\section{Repurposing Blu-ray movie discs as quasi-random nanoimprinting templates for photon management}

Alexander J. Smith ${ }^{1, \star}$, Chen Wang ${ }^{2, \star}$, Dongning Guo ${ }^{3}$, Cheng Sun ${ }^{2} \&$ Jiaxing Huang ${ }^{1}$

Quasi-random nanostructures have attracted significant interests for photon management purposes. To optimize such patterns, typically very expensive fabrication processes are needed to create the pre-designed, subwavelength nanostructures. While quasi-random photonic nanostructures are abundant in nature (for example, in structural coloration), interestingly, they also exist in Blu-ray movie discs, an already mass-produced consumer product. Here we uncover that Blu-ray disc patterns are surprisingly well suited for light-trapping applications. While the algorithms in the Blu-ray industrial standard were developed with the intention of optimizing data compression and error tolerance, they have also created quasi-random arrangement of islands and pits on the final media discs that are nearly optimized for photon management over the solar spectrum, regardless of the information stored on the discs. As a proof-of-concept, imprinting polymer solar cells with the Blu-ray patterns indeed increases their efficiencies. Simulation suggests that Blu-ray patterns could be broadly applied for solar cells made of other materials.

\footnotetext{
${ }^{1}$ Department of Materials Science and Engineering, Northwestern University, 2220 Campus Drive, Evanston, Illinois 60208, USA. ${ }^{2}$ Department of Mechanical Engineering, Northwestern University, 2145 Sheridan Road, Evanston, Illinois 60208, USA. ${ }^{3}$ Department of Electrical Engineering and Computer Science, Northwestern University, 2145 Sheridan Road, Evanston, Illinois 60208, USA. * These authors contributed equally to this work. Correspondence and requests for materials should be addressed to C.S. (email: c-sun@northwestern.edu) or to J.H. (email: jiaxing-huang@northwestern.edu).
} 
Q uasi-random nanostructures ${ }^{1-4}$ are abundant in nature for light manipulation purposes, such as in structural coloration in birds and insects ${ }^{5-7}$. These disordered structures have received renewed interest for photon management in a variety of engineering applications, such as highly efficient photon extraction in light-emitting diodes 8 , biomimetic structural coloration ${ }^{5,9}$ and random lasing ${ }^{10,11}$. Unlike perfectly periodic $^{12-16}$ or totally random structures, quasi-random nanostructures can offer both broadband absorption enhancement and customizable spectral response for different photoactive materials ${ }^{4,17-19}$. However, very expensive fabrication processes are typically needed to create these disordered, subwavelength patterns suitable for light trapping in photonic devices. Interestingly, such patterns already exist in a mass-produced consumer product.

Here we report repurposing of Blu-ray movie discs as quasirandom nanoimprinting templates. Regardless of the media's content, the audio and video compression algorithms convert the data to a high-entropy binary sequence. After error-correcting and modulation coding, a quasi-random pattern of pits is eventually generated on the disc, which we found to be surprisingly well suited for photon management over the entire solar spectrum. As a proof-of-concept, we successfully imprinted the Blu-ray pattern onto the active layer-and subsequently to the metal electrode-of polymer solar cells, leading to higher absorption and power conversion efficiencies. In addition, we numerically demonstrate the promise of this technique for enhancing light trapping for other photoactive materials. This new insight opens up promising areas for repurposing a low-cost consumer product for a high-end, value-added application.

\section{Results}

Assessing the Blu-ray pattern for light trapping. The arrangement of nanostructures within a light-trapping layer dramatically affects how photon energy is coupled into the plasmonic and waveguide modes confined in the active layer of a solar cell ${ }^{20}$. Figure 1 shows the Fourier transforms of subwavelength features arranged in periodic, random, quasi-random and Blu-ray patterns. The red and blue circles in Fourier space mark the $k$ values required to couple incoming light into the waveguide modes at the red and blue ends of the solar spectrum, respectively. Periodic (Fig. 1a) and random (Fig. 1b) patterns yield wavevectors that are either too discretized or too diffuse, respectively. On the other hand, quasi-random patterns (Fig. 1c) can be optimized to yield Fourier spectra that are efficient in light trapping, but these patterns are typically prohibitively expensive to manufacture. In comparison, although still containing a periodic component, the pattern on a pre-written Blu-ray movie disc (Fig. 1d) produces a close-to-optimized distribution of $k$-values.

The Blu-ray disc (BD) standard ${ }^{21}$ was developed for highdensity optical data storage, and has proven popular for distributing high-definition movies. The BD standard specifies that the track pitch and width are 320 and $130 \mathrm{~nm}$, respectively, and a single bit would be $75 \mathrm{~nm}$ long. When recorded to a disc, the video signals are first compressed to a binary sequence with high entropy per bit (for example, according to the MPEG-4 format), whose local statistics is are very similar to those of a sequence of random, independent, unbiased coin tosses ${ }^{22}$. When reading data from the disc, however, very short runs of successive zeros or ones yield low signal amplitudes and very long runs are difficult to distinguish from noise due to scratches or fingerprints ${ }^{21}$. Therefore, an error-control modulation code is applied to the data so that the number of successive zeros or ones is between 2 and 7 . When written to the disc, eventually a quasi-
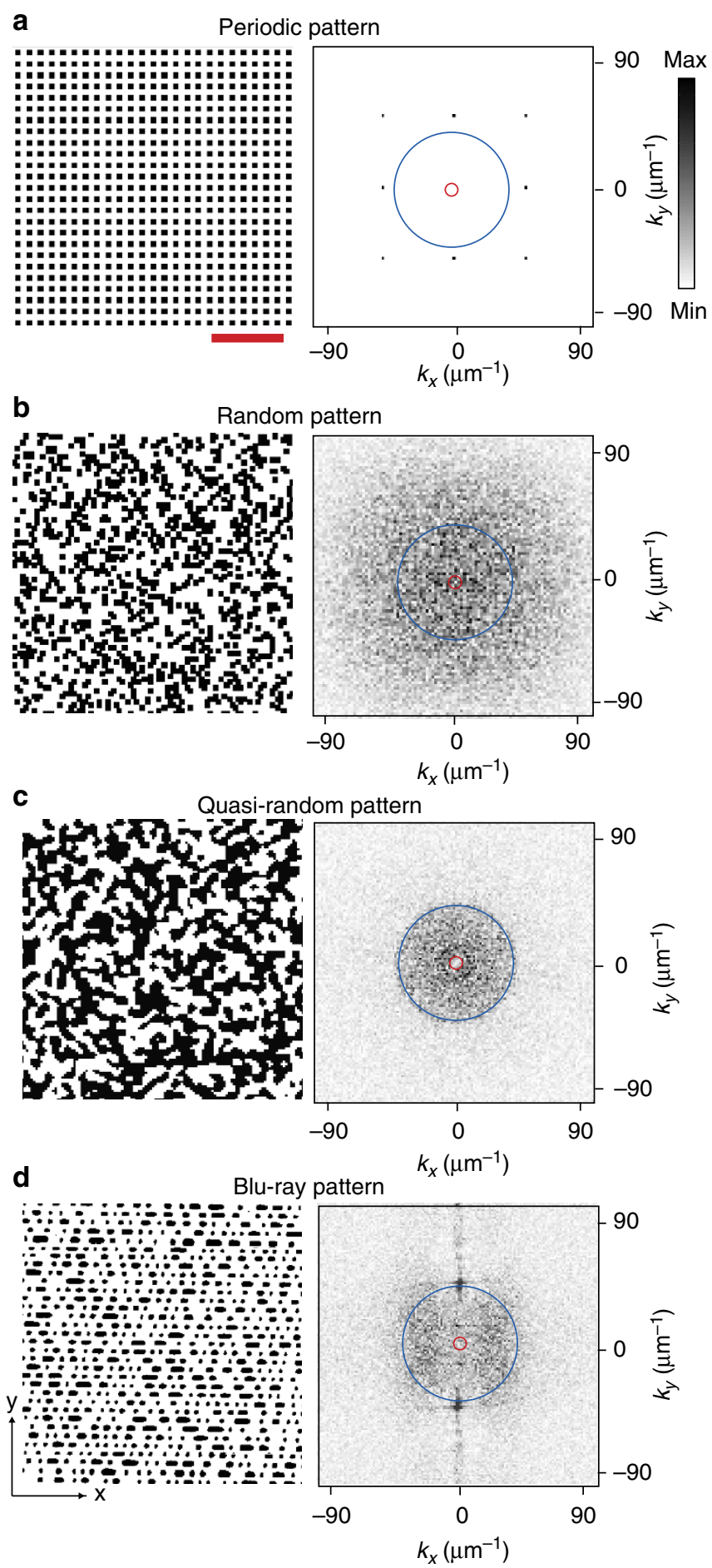

Figure 1 | Fourier transforms of subwavelength features arranged in periodic, random, quasi-random and Blu-ray patterns. Real space (red scale bar, $2.5 \mu \mathrm{m}$ ) and Fourier space. The circles denote the largest (blue) and smallest $k$-values needed to couple the entire solar spectrum ( $315 \mathrm{~nm}$ to $2.5 \mu \mathrm{m}$ ) to surface plasmons at the interface between an example photoactive layer, PTB7:PC 71 BM and silver electrode. (a) A periodic and (b) a random pattern yield wavevectors that are either too discretized or too diffuse to lie between the two circles, in contrast to (c) an optimized quasi-random pattern. (d) A Blu-ray pattern, obtained by thresholding an AFM image of the recording layer of a movie disc (see Supplementary Fig. 1), produces surprisingly close-to-optimized distribution of $k$-values. 
random pattern of islands and pits with lengths between 150 and $525 \mathrm{~nm}$ are generated (see a representative atomic force microscopy (AFM) image in Supplementary Fig. 1a), whose Fourier components are surprisingly well-suited for photon management over the broad visible and near-infrared portion of the spectrum, regardless of the movie content or area selected (Supplementary Fig. 2).

We numerically assessed the light-trapping effect of the Bluray structure on a typical polymer solar cell with a PTB7:PC ${ }_{71} B M$ active layer ${ }^{23}$ (see Supplementary Fig. 3a for the device structure) by performing one-dimensional (1D) rigorous coupled-wave analysis ${ }^{24}$. We modelled the absorption both parallel and perpendicular to the track, as marked by the four lines on the AFM image shown in Supplementary Fig. 3b. We calculated the corresponding absorption spectra from 315 to $775 \mathrm{~nm}$ both under transverse-electric (Supplementary Fig. 3c) and transverse-magnetic (Supplementary Fig. 3d) polarizations in reference to the plane of the cross-section. When compared to a non-patterned solar cell, the spectra of Blu-ray-patterned devices display broadband enhancement under both polarization conditions ${ }^{16,25,26}$. Extremely high enhancement $113.9 \%$ can be found in the region between 700 to $800 \mathrm{~nm}$ for transverse-magnetic polarization, indicating the underlying importance of the light-trapping effect in the weak absorption region of the active layer. The overall broadband absorption enhancement of a Blu-ray-patterned device, $18.2 \%$, was calculated by averaging over these simulation results, as shown in Supplementary Fig. 3e.

Photovoltaic device fabrication and characterization. Figure 2a illustrates the typical procedure for fabricating Blu-ray-patterned polymer solar cells. The BD was first delaminated to expose the pattern of pits and lands, which was replicated on a polydimethylsiloxane (PDMS) stamp. AFM image (Fig. 2b) of the resulting stamp confirms that it is a high-quality negative replica of the Blu-ray pattern with features as small as $150 \mathrm{~nm}$ across and $25 \mathrm{~nm}$ high. Next, the pattern was imprinted into a pre-fabricated polymer active layer by contact moulding using the stamp, followed by electrode deposition to complete the device. The final device structure is illustrated in Fig. 2c.

The AFM images in Fig. 3a clearly show successful transfer of the Blu-ray pattern to the active layer after nanoimprinting, in stark contrast with a non-patterned one. Line scans (Fig. 3a) show that the feature sizes are highly consistent with those of both the stamp and the original BD. The transferred pattern $\left(\sim 1 \mathrm{~cm}^{2}\right)$ displayed uniform iridescent reflection (Fig. 3b), demonstrating the reliability of this nanoimprinting process over large areas. We measured the reflection $(R)$ spectra of both non-patterned and Blu-ray-patterned solar cells and plotted $1-R$, that is, absorption in Fig. 3c. The absorption of the Blu-ray-imprinted cell is significantly enhanced by $21.8 \%$ over the entire absorption profile. Notably, the benefit of light trapping is most pronounced after $700 \mathrm{~nm}$, reaching $49.0 \%$, where the material absorbs weakly. Both observations are consistent with the simulation shown in Supplementary Fig. 3. The enhanced broadband absorption of Blu-ray-patterned solar cells indeed led to improved external quantum efficiencies (EQEs) (Fig. 3d). The overall EQE enhancement averaged over the entire absorption profile is $30.8 \%$, while the averaged enhancement is $85.0 \%$ for wavelengths $>700 \mathrm{~nm}$. As a result, the patterned cells delivered $16.9 \%$ higher short-circuit current densities $\left(J_{\mathrm{sc}}\right)$, eventually leading to a power conversion efficiency enhancement of $11.9 \%$ (see Supplementary Fig. 4 and Supplementary Table 1 for detailed performance results). Although higher $J_{\mathrm{sc}}$ values have been reported for PTB7:PC ${ }_{71}$ BM solar cells with thicker active layers $(\sim 100 \mathrm{~nm})^{23}$, in the current study, an active layer thickness of $50 \mathrm{~nm}$ was chosen to best demonstrate the light-trapping effect while avoiding shorting caused by imprinting with the 25-nm-deep pattern. However, the depth of the pits could be modified during the mould fabrication step (for example, via etching) to accommodate the needs of alternative thicknesses for device optimization.

\section{Discussion}

The work here demonstrated the use of pre-written, Blu-ray movie discs as extremely low-cost nanoimprinting templates for creating photon management nanostructures in thin-film solar cells. Mode analysis (Supplementary Fig. 5) suggests that the Blu-ray patterns can be broadly applied for light trapping in other types of solar cells, including those made with amorphous Si, III-V and II-VI semiconductors, and perovskite compounds. In contrast to previous attempts to make use of the periodic micro/nanostructures in blank optical storage media $^{27-29}$, here we take advantage of the quasi-random patterns in information-laden discs. Thus the new insight opens up promising areas, where repurposing of a low-cost consumer product leads to much higher-end, value-added applications. a

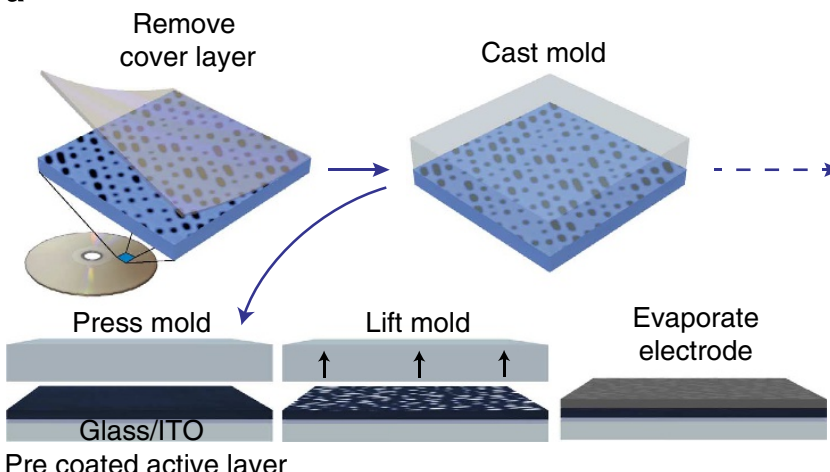

b

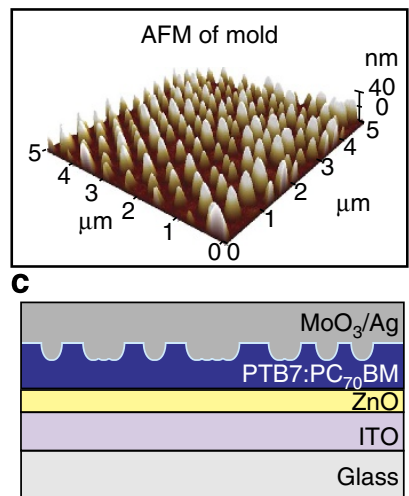

Figure 2 | Processing of Blu-ray-patterned solar cells. (a) Schematic diagramming the process for delaminating a BD and casting a PDMS mould on the exposed recording layer; fabricating the nanopatterned solar cells; and imprinting the active layer using the nanopatterned PDMS mould and evaporating the $\mathrm{MoO}_{3} / \mathrm{Ag}$ electrode. (b) AFM image of the nanopatterned PDMS mould. (c) Schematic diagram of the solar cell architecture used in the numerical and experimental portions of this work. 
a

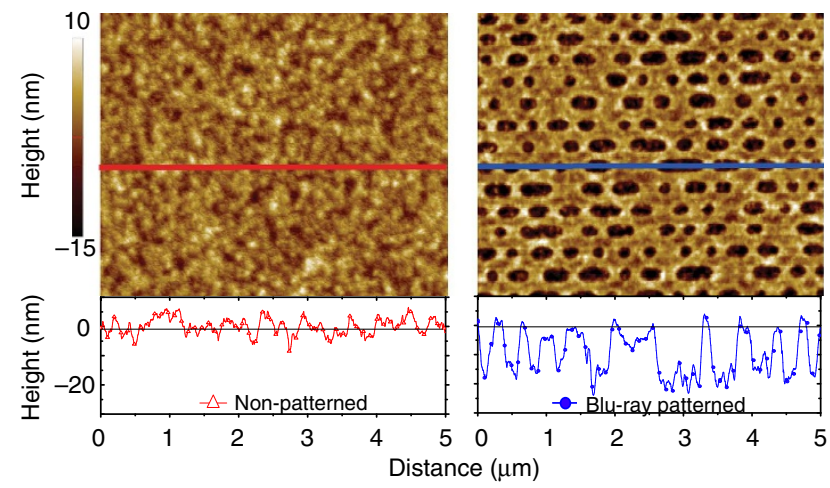

b

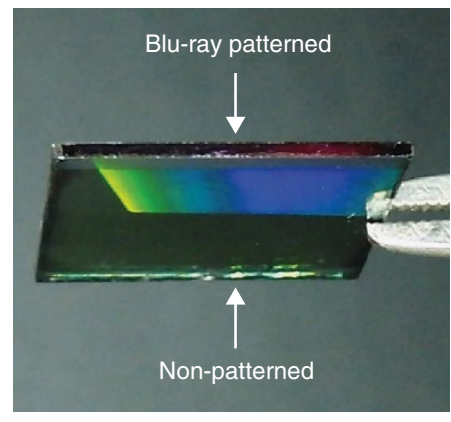

C

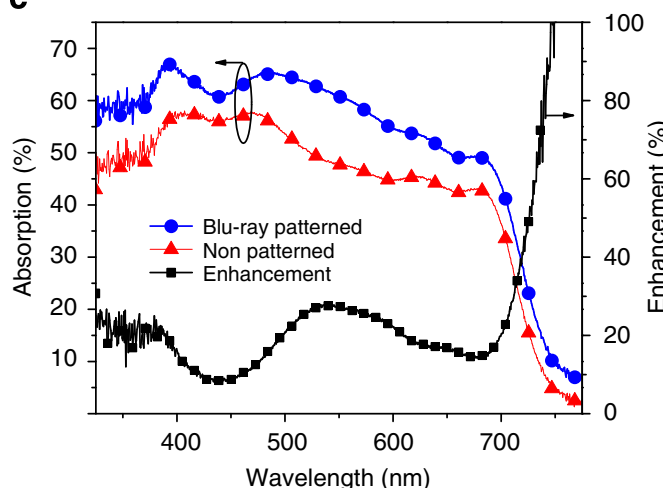

d

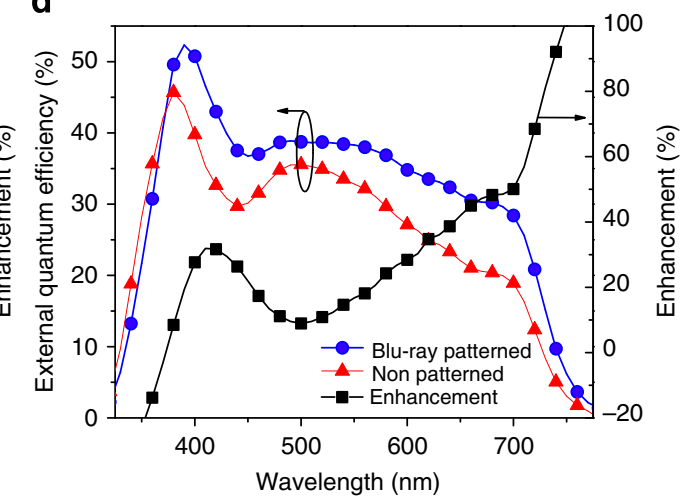

Figure 3 | Physical morphology and performance characteristics of Blu-ray-patterned solar cells. (a) AFM images of the active layer without stamping and after stamping with a Blu-ray-patterned mould. The horizontal line profiles illustrate the depth contrast of the Blu-ray-patterned active layer. (b) Optical images of a half-patterned solar cell showing iridescent scattering due to the periodic nature of the Blu-ray pattern. (c) Reflected absorption (1-R) measurements from a Blu-ray-patterned solar cell. (d) Measured EQEs show a broadband enhancement and agree well with the calculated absorption spectrum in (c).

\section{Methods}

Fourier transforms. We computed the two-dimensional (2D) discrete Fourier transform of the images in Fig. 1 (left) and customized the colour map to improve the contrast of the Fourier transform (FT) images so as to better compare the features.

Numerical calculations. The absorption was calculated by rigorous coupled-wave analysis, which is one of the most commonly used techniques to solve the scattering problem in Fourier space. For the multilayered dielectric stacks, Fourier expansions of both the field and the permittivity lead to an algebraic eigenvalue system for each layer. As we are interested in quasi-randomness along one direction, a $1 \mathrm{D}$ simulation is implemented with periodicity $10 \mu \mathrm{m}$ The number of Fourier components considered is 289 and the convergence test was performed on the selection of the diffraction order to ensure the numerical accuracy.

Nanopatterned mould fabrication. We fabricated PDMS nanopatterned moulds using a delaminated $\mathrm{BD}$ as the master. We first trimmed the Blu-ray movie disc (Police Story 3: Super Cop) around the edges using scissors, and then carefully peeled off the cover layer (Fig. 1a) to reveal the pattern shown in Supplementary Fig. 1. No cleaning or secondary processing of the $\mathrm{BD}$ was required, as the cover layer effectively protects the nanopatterned surface after manufacturing. We then cast PDMS on top of the delaminated $\mathrm{BD}$ and cured it overnight at $60^{\circ} \mathrm{C}$. After preparation, the PDMS mould could be lifted off the $\mathrm{BD}$ and reused several times. It should be noted that the cover layer, which we removed as shown in Fig. 2a, contains the same pattern as the PDMS mould and can also be used as a nanoimprinting stamp. However, we found that PDMS stamps could more reliably transfer the $2 \mathrm{D}$ pattern to polymer thin films due to their flexibility, ductility and favourable surface energy properties.

Device fabrication. Figure 2 schematically shows the device fabrication procedure. We fabricated all devices on patterned ITO-coated glass substrates $\left(20 \Omega \mathrm{sq}^{-1}\right)$ that were pre-cleaned and treated with oxygen plasma immediately before use. We employed a sol-gel synthesis for the ZnO ETL following ref. 30. We spin-coated $0.25 \mathrm{M}$ zinc acetate dihydrate and $0.25 \mathrm{M}$ ethanolamine in 2-methoxyethanol through a polyvinylidene difluoride filter and annealed at $180^{\circ} \mathrm{C}$ for $20 \mathrm{~min}$ to yield a 15-nm-thick film. We verified the $15 \mathrm{~nm}$ thickness of the $\mathrm{ZnO}$ films using spectroscopic ellipsometry (J.A. Woollam M2000U). We prepared the active material by blending PTB7 (1-material) with $\mathrm{PC}_{71} \mathrm{BM}$ (nano-C) at 1.0:1.5 in a solution of $97 \mathrm{vol} \%$ chlorobenzene (Sigma) and 3 vol\% diiodooctane (Sigma) and mixed for 2 days at $65^{\circ} \mathrm{C}$. A solution concentration of $10 \mathrm{mg} \mathrm{ml}^{-1}$ by total weight yielded an active layer thickness of $50 \mathrm{~nm}$. After spin-coating, we gently placed a PDMS stamp on top of the active layer (for stamped devices) and placed all devices under vacuum for $2 \mathrm{~h}$ in order to allow for evaporation of the diiodooctane, and for $\mathrm{PTB} 7$ and $\mathrm{PC}_{71} \mathrm{BM}$ domains to phase segregate. It should be noted that if the stamp was removed immediately the nanopattern would fade away within a few hours, which we attribute to the residual solvent allowing the active material to reconfigure into a lower-energy (that is, flatter) morphology. After carefully removing the stamp, all devices were moved to a thermal evaporator (Angstron Covap II), where we deposited $10 \mathrm{~nm}$ of $\mathrm{MoO}_{3}$ (Alfa Aesar, Puratronic, 99.9995\%) and $100 \mathrm{~nm}$ of Ag. We also conducted control experiments with devices stamped with a flat PDMS mould. The physical morphologies of the flat-stamped control and non-stamped active layers are comparable (Supplementary Fig. 6), with the flat-stamped device being slightly smoother.

Characterization of photovoltaic cells. We performed microscopic and morphological characterization using a scanning electron microscope (FEI Nova 600) and AFM (Park Systems XE-100), respectively. We obtained reflection data with an Andor SR-303i_A spectrometer combined with Leica DMI $3000 \mathrm{M}$ microscope $(10 \times$ objective, numerical aperture $=0.3,100 \mathrm{~W}$ halogen light source $)$. We measured current density-voltage characteristics of all devices under AM1.5 G illumination using an Oriel Xe solar simulator, employing filters to cut off grating overtones. Correct current density measurements were calculated after measuring EQE under short-circuit conditions (Enli Technology QE-R3018). A calibrated monosilicon diode with known spectral response served as a reference. We averaged over the best 12 devices measured to obtain our device statistics. 


\section{References}

1. Vardeny, Z. V., Nahata, A. \& Agrawal, A. Optics of photonic quasicrystals. Nat. Photon. 7, 177-187 (2013).

2. Wiersma, D. S. Disordered photonics. Nat. Photon. 7, 188-196 (2013).

3. Vynck, K., Burresi, M., Riboli, F. \& Wiersma, D. S. Photon management in twodimensional disordered media. Nat. Mater. 11, 1017-1022 (2012).

4. Martins, E. R. et al. Deterministic quasi-random nanostructures for photon control. Nat. Commun. 4, 2665 (2013).

5. Vukusic, P., Hallam, B. \& Noyes, J. Brilliant whiteness in ultrathin beetle scales. Science 315, 348-348 (2007).

6. Dong, B. Q. et al. Optical response of a disordered bicontinuous macroporous structure in the longhorn beetle Sphingnotus mirabilis. Phys. Rev. E 84, 011915 (2011).

7. Dufresne, E. R. et al. Self-assembly of amorphous biophotonic nanostructures by phase separation. Soft Matter 5, 1792-1795 (2009).

8. Koo, W. H. et al. Light extraction from organic light-emitting diodes enhanced by spontaneously formed buckles. Nat. Photon. 4, 222-226 (2010).

9. Forster, J. D. et al. Biomimetic isotropic nanostructures for structural coloration. Adv. Mater. 22, 2939-2944 (2010).

10. Conley, G. M., Burresi, M., Pratesi, F., Vynck, K. \& Wiersma, D. S. Light transport and localization in two-dimensional correlated disorder. Phys. Rev. Lett. 112, 143901 (2014).

11. Noh, H. et al. Control of lasing in biomimetic structures with short-range order. Phys. Rev. Lett. 106, 183901 (2011).

12. Garnett, E. \& Yang, P. D. Light trapping in silicon nanowire solar cells. Nano Lett. 10, 1082-1087 (2010).

13. Zhan, Y. H., Li, X. F. \& Li, Y. Numerical simulation of light-trapping and photoelectric conversion in single nanowire silicon solar cells. IEEE J. Sel. Top. Quant. Electron. 19, 4000208 (2013).

14. Raman, A., Yu, Z. F. \& Fan, S. H. Dielectric nanostructures for broadband light trapping in organic solar cells. Opt. Express 19, 19015-19026 (2011).

15. Wang, K. X. Z., Yu, Z. F., Liu, V., Cui, Y. \& Fan, S. H. Absorption enhancement in ultrathin crystalline silicon solar cells with antireflection and light-trapping nanocone gratings. Nano Lett. 12, 1616-1619 (2012).

16. You, J. B. et al. Surface plasmon and scattering-enhanced low-bandgap polymer solar cell by a metal grating back electrode. Adv. Energy Mater. 2, 1203-1207 (2012).

17. Wang, C., Yu, S. C., Chen, W. \& Sun, C. Highly efficient light-trapping structure design inspired by natural evolution. Sci. Rep. 3, 1025 (2013)

18. Pala, R. A. et al. Optimization of non-periodic plasmonic light-trapping layers for thin-film solar cells. Nat. Commun 4, 2095 (2013).

19. Ferry, V. E. et al. Optimized spatial correlations for broadband light trapping nanopatterns in high efficiency ultrathin film a-Si:H solar cells. Nano Lett. 11, 4239-4245 (2011)

20. Atwater, H. A. \& Polman, A. Plasmonics for improved photovoltaic devices. Nat. Mater. 9, 865-865 (2010).

21. Blu-ray Disc Association. Blu-ray Disc Format. White Paper, 3rd edn (Blu-ray Disc Association, 2012).

22. Visweswariah, K., Kulkarni, S. R. \& Verdú, S. Source codes as random number generators. IEEE Trans. Inf. Theory 44, 462-471 (1998).

23. Liang, Y. Y. \& Yu, L. P. A new class of semiconducting polymers for bulk heterojunction solar cells with exceptionally high performance. Acc. Chem. Res. 43, 1227-1236 (2010)
24. Li, L. F. Formulation and comparison of two recursive matrix algorithms for modeling layered diffraction gratings. J. Opt. Soc. Am. A 13, 1024-1035 (1996).

25. Battaglia, C. et al. Light trapping in solar cells: can periodic beat random? ACS Nano 6, 2790-2797 (2012).

26. Brongersma, M. L., Cui, Y. \& Fan, S. H. Light management for photovoltaics using high-index nanostructures. Nat. Mater. 13, 451-460 (2014).

27. Yu, H. Z., Li, Y. C. \& Ou, L. M. L. Reading disc-based bioassays with standard computer drives. Acc. Chem. Res. 46, 258-268 (2013).

28. Baba, A., Wakatsuki, K., Shinbo, K., Kato, K. \& Kaneko, F. Increased shortcircuit current in grating-coupled surface plasmon resonance field-enhanced dye-sensitized solar cells. J. Mater. Chem. 21, 16436-16441 (2011).

29. Baba, A., Aoki, N., Shinbo, K., Kato, K. \& Kaneko, F. Grating-coupled surface plasmon enhanced short-circuit current in organic thin-film photovoltaic cells. ACS Appl. Mater. Inter. 3, 2080-2084 (2011).

30. Luck, K. A. et al. Improved uniformity in high-performance organic photovoltaics enabled by ( 3 -aminopropyl) triethoxysilane cathode functionalization. Phys. Chem. Chem. Phys. 15, 20966-20972 (2013).

\section{Acknowledgements}

This work was primarily funded by the National Science Foundation through CCF 1423040 (D.G.), CMMI-CAREER 0955195, CMMI-1130640 (C.S.) and CMMI 1130407 (J.H.). A.J.S. acknowledges the Northwestern University International Institute for Nanotechnology for a Ryan Fellowship. J.H. acknowledges the John Simon Guggenheim Memorial Foundation for a Guggenheim Fellowship, the Sony Corporation for a gift donation and Dr. Shaorong Liu from IBM for initial discussions on the data compression algorithm. The work was made possible through the use of facilities in the newly founded MSE Energy Materials Laboratory at Northwestern University, which was partially supported by a donation from Mr and Mrs Meister, an award from the Walter P. Murphy Society and Northwestern MRSEC (NSF DMR-1121262). We thank Professor S.I. Stupp for letting us use his glove boxes.

\section{Author contributions}

A.J.S. initiated the work through discussions with C.W., C.S. and J.H., and conducted device fabrication and characterization. C.W. conducted all numerical simulations and Fourier transforms, performed all light absorption measurements and assisted with device characterization. J.H. first conjectured that Blu-ray patterns could be universally quasi-random regardless of disc contents. D.G. improved the other authors' understanding of the Blu-ray compression algorithms. All authors contributed to the writing of the manuscript.

\section{Additional information}

Supplementary Information accompanies this paper at http://www.nature.com/ naturecommunications

Competing financial interests: The authors declare no competing financial interests.

Reprints and permission information is available online at http://npg.nature.com/ reprintsandpermissions/

How to cite this article: Smith, A. J. et al. Repurposing Blu-ray movie discs as quasi-random nanoimprinting templates for photon management. Nat. Commun. 5:5517 doi: $10.1038 /$ ncomms6517 (2014). 